\title{
Association between Smoking and Liver Fibrosis among Patients with Nonalcoholic Fatty Liver Disease
}

\author{
Hongjie Ou, ${ }^{1}$ Yaojie Fu, ${ }^{2}$ Wei Liao $\left(\mathbb{D},{ }^{3,4}\right.$ Caixia Zheng, ${ }^{1}$ and Xiaolu Wu ${ }^{1}{ }^{1}$ \\ ${ }^{1}$ Department of Infectious Diseases, First Affiliated Hospital of Xiamen University, Xiamen, Fujian, China \\ ${ }^{2}$ Emergency Department, First Affiliated Hospital of Xiamen University, Xiamen, Fujian, China \\ ${ }^{3}$ Intensive Care Unit, Sun Yat-sen University Cancer Center, Guangzhou, China \\ ${ }^{4}$ Sun Yat-sen University Cancer Center, State Key Laboratory of Oncology in South China, \\ Collaborative Innovation Center for Cancer Medicine, Guangzhou, China
}

Correspondence should be addressed to Wei Liao; liaowei@sysucc.org.cn

Received 22 July 2019; Accepted 12 September 2019; Published 15 October 2019

Guest Editor: Roberto Martínez-Beamonte

Copyright (c) 2019 Hongjie Ou et al. This is an open access article distributed under the Creative Commons Attribution License, which permits unrestricted use, distribution, and reproduction in any medium, provided the original work is properly cited.

Objective. We aimed at analyzing the role of smoking in hepatic fibrosis in patients with nonalcoholic fatty liver disease (NAFLD) and at exploring the related risk factors. Methods. This was a cross-sectional study that included a total of 225 patients with NAFLD. Among them, 127 were nonsmokers and 98 were smokers. Liver significant fibrosis was diagnosed when the liver stiffness (LS) value was higher than $7.4 \mathrm{kPa}$. The diagnostic criterion for NAFLD was a controlled attenuation parameter (CAP) value of $>238 \mathrm{~dB} / \mathrm{m}$. The CAP and LS values were measured using FibroScan. Results. FibroScan showed that the LS value in the smokers was significantly higher than that in the nonsmokers $(10.12 \pm 10.38 \mathrm{kPa}$ vs. $7.26 \pm 6.42 \mathrm{kPa}, P=0.013)$. The proportions of patients with liver significant fibrosis and advanced liver fibrosis among the smokers were significantly higher than those among the nonsmokers $(P=0.046)$. Univariate analysis showed that age, weight, high AST level, low PLT level, and smoking were the risk factors associated with liver fibrosis in the smokers with NAFLD while multivariate analysis showed that age $(\mathrm{OR}=1.029$, $P=0.021)$, high AST level $(\mathrm{OR}=1.0121, P=0.025)$, and smoking $(\mathrm{OR}=1.294, P=0.015)$ were the independent risk factors associated with liver fibrosis in the patients with NAFLD. Moreover, high AST level $(\mathrm{OR}=1.040, P=0.029)$, smoking index $(\mathrm{OR}=1.220, P=0.019)$, and diabetes mellitus $(\mathrm{OR}=1.054, P=0.032)$ were the independent risk factors for liver fibrosis among the smokers with NAFLD. Conclusion. This study showed that smoking was closely associated with liver fibrosis among the patients with NAFLD. For patients with NAFLD who smoke, priority screening and timely intervention should be provided if they are at risk of liver fibrosis.

\section{Introduction}

With the increase in the incidence of obesity and the associated metabolic syndrome, nonalcoholic fatty liver disease (NAFLD) has become an important cause of chronic liver disease [1]. Epidemiological studies have shown that $20 \%$ to $30 \%$ of individuals in Western countries develop NAFLD [1-3]. According to the definition of the American Association for the Study of Liver Diseases, NAFLD is a disease characterized by hepatic steatosis and lipid storage without excessive drinking history [2]. According to the change in the pathological degree, NAFLD can be divided into three stages: simple fatty liver (nonalcoholic fatty liver), nonalcoholic steatohepatitis, and cirrhosis.

NAFLD is closely related to metabolic syndrome [4]. Obesity, type 2 diabetes mellitus, and dyslipidemia are considered to be important risk factors for NAFLD $[1,4-6]$. NAFLD is closely related not only to metabolic abnormalities but also to poor living behaviors [7-9]. All three abovementioned important risk factors for NAFLD are associated with unhealthy lifestyles. Therefore, NAFLD is generally considered to be a disease associated with an unhealthy lifestyle. Many studies have revealed that changes in unhealthy lifestyles can reduce the transaminase levels and improve NAFLD [10-13]. 
Smoking is a common poor living behavior in daily life. It can damage the antioxidant system [14, 15]. Although smoking can increase the risk for liver fibrosis and cirrhosis in patients with chronic hepatitis $\mathrm{B}(\mathrm{CHB})$ infection $[16,17]$, only a few studies have investigated the relationship between smoking and NAFLD. Suzuki et al. [18] reported that smoking is associated with high levels of alanine aminotransferase (ALT) in patients with NAFLD. Another study reported that smoking is an independent risk factor for NAFLD [19]. Although these authors have confirmed that smoking is associated with the occurrence of NAFLD, there is no research report on whether smoking promotes liver fibrosis in patients with NAFLD.

Hence, we enrolled smokers and nonsmokers with NAFLD and analyzed liver fibrosis among the smokers in this study. The risk factors for liver fibrosis were explored to provide medical evidence for screening and early diagnosis of liver fibrosis in smokers with NAFLD.

\section{Methods}

2.1. Subjects. This was a cross-sectional study that included a total of 225 patients with NAFLD. Among them, 127 were nonsmokers and 98 were smokers. All patients were recruited from the First Affiliated Hospital of Xiamen University from May 2015 to April 2018. Patients were included when they met the following criteria: diagnosis of NAFLD according to the diagnostic criterion of a controlled attenuation parameter (CAP) value of $>238 \mathrm{~dB} / \mathrm{m}$ according to previous recommendations and confirmation with ultrasonography $[4,20-22]$. Conversely, patients were excluded when they met the following criteria: (1) use of medications that can induce hepatic steatosis (e.g., corticosteroids, estrogen, methotrexate, or amiodarone) within 6 months of study inclusion, (2) evidence of coinfection with hepatitis $\mathrm{C}$, hepatitis $\mathrm{D}$, or human immunodeficiency virus, (3) autoimmune liver disease, and (4) heavy alcohol consumption or alcohol abuse, defined as alcohol consumption of $>10 \mathrm{~g} /$ day. The Institutional Review Board of the First Affiliated Hospital of Xiamen University approved the study. Each enrolled patient provided informed consent.

2.2. FibroScan Test. Liver fibrosis and steatosis were diagnosed on the basis of the liver stiffness (LS) and CAP values $[23,24]$. These values were assessed by a professionally trained technician using FibroScan (Echosens, Paris, France) according to the manufacturer's instructions. The LS values were expressed in kilopascals and CAP values in decibels per meter. The ratio of the interquartile range (IQR) of the LS value to the median (IQR/M) was calculated as an indicator of variability. Only procedures with at least 10 valid measurements, a success rate of at least $60 \%$, and an IQR/M ratio of $<0.3$ were considered reliable and then used for the analysis. The CAP value was measured only using validated measurement tools according to the same criteria used for the LS value and on the same signals, ensuring obtainment of a liver ultrasonic attenuation simultaneously and in the same volume of liver parenchyma as in the LS value. The median of the individual measurements was considered the final CAP value.

Among the patients with NAFLD, hepatic steatosis was diagnosed at the CAP values of $>238 \mathrm{~dB} / \mathrm{m}$, according to previous recommendations [4, 20-22].

2.3. Patient Information Collection. Patient information, including demographic characteristics, physical examination, and laboratory test results, was collected. The demographic characteristics assessed included age, sex, and smoking history. Physical examination results, including height and weight, were recorded. Blood pressure was also measured after the FibroScan test. Laboratory test results, including the levels of platelet (PLT), serum aspartate aminotransferase (AST), and ALT, were collected in accordance with standard procedures. These laboratory test results were obtained using standard automated techniques within 14 days of the FibroScan test. Smoking index = daily tobacco intake $*$ duration of smoking.

Blood pressure was measured using a standard mercury sphygmomanometer. All patients were asked to rest for at least 5 minutes before measurement. Each patient required at least three blood pressure measurements, with an interval of 1 minute each. The average value of the three measurements was used for the analysis.

2.4. Statistical Analysis. Continuous variables were expressed as means \pm standard deviations and categorical variables as percentages. The chi-square test and $t$-test were used to detect whether differences between the two groups were statistically significant. Univariate and multivariate logistic regression analyses were used to explore the risk factors associated with liver fibrosis and advanced liver fibrosis in the patients with NAFLD. The Data Analysis and Quality Control Program for SPSS for Windows version 13.0 was applied for the statistical analysis.

\section{Results}

3.1. Demographic and Clinical Characteristics of the Patients. A total of 225 patients with NAFLD were enrolled in this study. Among these patients, 98 were smokers (smoking group) and 127 were nonsmokers (nonsmoking group). The proportion of male patients in the smoking group was significantly higher than that in the nonsmoking group $(P<0.001)$. The weight of the patients in the smoking group was higher than that of the patients in the nonsmoking group $(P=0.037)$. The proportion of patients with diabetes mellitus in the nonsmoking group was significantly lower than that in the smoking group $(P=0.012)$. The serum levels of ALT, AST, and PLT were comparable between the two groups, as shown in Table 1.

3.2. Comparison of Liver Fibrosis between the Two Groups. The liver conditions of fibrosis were compared (Table 2). The LS value of the smoking group was significantly higher than that of the nonsmoking group $(10.12 \pm 10.38 \mathrm{kPa}$ vs. 
TABle 1: Baseline demographic and clinical characteristics by groups.

\begin{tabular}{lccc}
\hline \multirow{2}{*}{ Variables } & \multicolumn{2}{c}{ Nonalcoholic fatty liver disease } & \\
& Smoking & Nonsmoking & $P$ \\
\hline Sample size & 98 & 127 & \\
Sex (F/M) & $3 / 95$ & $49 / 78$ & $<0.001$ \\
Age (years) & $45.77 \pm 13.12$ & $41.01 \pm 11.55$ & 0.004 \\
Height $(\mathrm{cm})$ & $168.36 \pm 6.47$ & $166.96 \pm 7.48$ & 0.142 \\
Weight $(\mathrm{kg})$ & $67.53 \pm 12.50$ & $63.98 \pm 12.76$ & 0.037 \\
SBP $(\mathrm{mm} / \mathrm{Hg})$ & $127.03 \pm 12.52$ & $129.09 \pm 14.15$ & 0.260 \\
DBP $(\mathrm{mm} / \mathrm{Hg})$ & $82.07 \pm 7.85$ & $83.66 \pm 9.28$ & 0.177 \\
ALT & $90.74 \pm 72.34$ & $80.49 \pm 81.94$ & 0.324 \\
AST & $67.84 \pm 45.29$ & $63.41 \pm 40.67$ & 0.444 \\
PLT & $216.11 \pm 63.96$ & $210.11 \pm 60.91$ & 0.476 \\
T2DM (Y/N) & $21 / 77$ & $12 / 115$ & 0.012 \\
\hline
\end{tabular}

TABle 2: Proportion of liver fibrosis and advanced fibrosis by groups.

\begin{tabular}{|c|c|c|c|}
\hline \multirow[t]{2}{*}{ Variables } & \multicolumn{2}{|c|}{$\begin{array}{c}\text { Nonalcoholic fatty liver } \\
\text { disease }\end{array}$} & \multirow[t]{2}{*}{$P$} \\
\hline & Smoking & Nonsmoking & \\
\hline Sample size & 98 & 127 & \\
\hline Liver stiffness value & & & 0.046 \\
\hline$<7.4(\mathrm{kPa})$ & 52 & 88 & \\
\hline $7.4-9.8(\mathrm{kPa})$ & 22 & 16 & \\
\hline$>9.8(\mathrm{kPa})$ & 24 & 23 & \\
\hline Liver stiffness value, $\mathrm{kPa}$ & $10.12 \pm 10.38$ & $7.26 \pm 6.42$ & 0.013 \\
\hline
\end{tabular}

$7.26 \pm 6.42 \mathrm{kPa}, P=0.013)$. The proportion of patients with liver significant fibrosis and advanced fibrosis in the smoking group was significantly higher than that in the nonsmoking group $(P=0.046)$.

3.3. Risk Factors Associated with Fibrosis in the Patients with NAFLD. The univariate and multivariate analyses were conducted to explore the risk factors associated with fibrosis among the patients with NAFLD. The results are shown in Table 3. The univariate analysis showed that age, weight, high AST level, low PLT level, and smoking were the risk factors associated with liver fibrosis in the smokers with NAFLD. Conversely, the multivariate analysis showed that age $(\mathrm{OR}=1.029, P=0.021)$, high $\mathrm{AST}$ level $(\mathrm{OR}=1.0121$, $P=0.025)$, and smoking $(\mathrm{OR}=1.294, P=0.015)$ were the independent risk factors associated with liver fibrosis in the patients with NAFLD.

3.4. Clinical Characteristics of the Patients with NAFLD with and without Liver Fibrosis. To analyze the related factors for liver fibrosis in the smokers with NAFLD further, we subdivided the smokers into the fibrosis and nonfibrosis subgroups. The clinical characteristics of these two subgroups are shown in Table 4 . The average age $(P=0.032)$ and AST level $(P=0.001)$ in the fibrosis group were significantly higher than those in the nonfibrosis group, while the PLT level was lower in the fibrosis group than in the nonfibrosis group $(P=0.036)$. In addition, the proportion of
TABLE 3: Risk factors associated with fibrosis in NAFLD patients.

\begin{tabular}{lcccccc}
\hline \multirow{2}{*}{ Variables } & \multicolumn{3}{c}{ Univariate analysis } & \multicolumn{3}{c}{ Multivariate analysis } \\
& OR & $95 \%$ CI & $P$ & OR & $95 \%$ CI & $P$ \\
\hline Sex & 1.622 & $0.366-3.322$ & 0.632 & & & \\
Age & 1.022 & $1.006-1.097$ & 0.019 & 1.029 & $1.004-1.055$ & 0.021 \\
Height & 0.936 & $0.875-1.001$ & 0.055 & & & \\
Weight & 1.066 & $1.028-1.107$ & 0.001 & & & \\
SBP & 1.014 & $0.958-1.073$ & 0.632 & & & \\
DBP & 0.922 & $0.842-1.009$ & 0.076 & & & \\
ALT & 0.998 & $0.994-1.002$ & 0.339 & & & \\
AST & 1.009 & $1.001-1.017$ & 0.020 & 1.012 & $1.002-1.061$ & 0.025 \\
PLT & 0.991 & $0.985-0.996$ & 0.001 & & & \\
Smoking & 1.305 & $1.152-2.611$ & 0.011 & 1.294 & $1.087-2.087$ & 0.015 \\
index & & & & & & \\
T2DM & 1.022 & $0.998-1.525$ & 0.062 & & & \\
\hline
\end{tabular}

TABLE 4: Characteristics of NAFLD patients with smoking with or without liver fibrosis.

\begin{tabular}{|c|c|c|c|}
\hline \multirow[t]{2}{*}{ Variables } & \multicolumn{2}{|c|}{$\begin{array}{l}\text { Nonalcoholic fatty liver disease } \\
\text { with smoking }\end{array}$} & \multirow[t]{2}{*}{$P$} \\
\hline & Fibrosis & Nonfibrosis & \\
\hline Sample size & 46 & 52 & \\
\hline $\operatorname{Sex}(F / M)$ & $1 / 45$ & $2 / 50$ & 0.632 \\
\hline Age (years) & $48.78 \pm 11.65$ & $43.09 \pm 13.92$ & 0.032 \\
\hline Height $(\mathrm{cm})$ & $168.17 \pm 6.51$ & $168.52 \pm 6.49$ & 0.794 \\
\hline Weight (kg) & $67.37 \pm 13.57$ & $65.79 \pm 11.56$ & 0.535 \\
\hline $\mathrm{SBP}(\mathrm{mm} / \mathrm{Hg})$ & $125.71 \pm 14.25$ & $128.17 \pm 10.82$ & 0.337 \\
\hline $\mathrm{DBP}(\mathrm{mm} / \mathrm{Hg})$ & $80.76 \pm 8.92$ & $83.21 \pm 6.64$ & 0.124 \\
\hline ALT & $87.91 \pm 82.58$ & $72.12 \pm 81.30$ & 0.344 \\
\hline AST & $83.78 \pm 38.15$ & $53.74 \pm 46.76$ & 0.001 \\
\hline PLT & $201.55 \pm 70.21$ & $228.99 \pm 55.43$ & 0.036 \\
\hline $\mathrm{T} 2 \mathrm{DM}(\mathrm{Y} / \mathrm{N})$ & $14 / 32$ & $7 / 45$ & 0.041 \\
\hline Smoking index & $583.26 \pm 480.72$ & $388.63 \pm 458.52$ & 0.043 \\
\hline
\end{tabular}

patients with diabetes mellitus in the fibrosis group was significantly higher than that in the nonfibrosis group $(P=0.041)$; the smoking index was significantly higher in the fibrosis group than in the nonfibrosis group $(P=0.043)$.

3.5. Risk Factors Associated with Fibrosis in Smokers with NAFLD. We further analyzed the factors associated with liver fibrosis in the smokers with NAFLD, and the results are shown in Table 5. The univariate analysis showed that age, high AST level, low PLT level, smoking index, and diabetes mellitus were the risk factors for fibrosis among these patients. Conversely, the multivariate analysis indicated that high AST level $(\mathrm{OR}=1.040, P=0.029)$, smoking index $(\mathrm{OR}=1.220, P=0.019)$, and diabetes mellitus $(\mathrm{OR}=1.054$, $P=0.032$ ) were the independent risk factors for liver fibrosis among them.

\section{Discussion}

In this study, we confirmed that smoking is closely associated with NAFLD. Moreover, we further confirmed that it is closely related to liver fibrosis in NAFLD. The LS value of the smokers with NAFLD was significantly higher than that of the nonsmokers with NAFLD. Older age, high AST level, 
TABLE 5: Risk factors for fibrosis in NAFLD patients with smoking.

\begin{tabular}{lcccccc}
\hline \multirow{2}{*}{ Variables } & \multicolumn{3}{c}{ Univariate analysis } & \multicolumn{3}{c}{ Multivariate analysis } \\
& OR & $95 \%$ CI & $P$ & OR & $95 \%$ CI & $P$ \\
\hline Sex & 1.022 & $0.395-1.895$ & 0.614 & & & \\
Age & 1.049 & $1.004-1.095$ & 0.029 & & & \\
Height & 0.956 & $0.867-1.054$ & 0.983 & & & \\
Weight & 1.069 & $1.007-1.136$ & 0.176 & & & \\
SBP & 1.036 & $0.943-1.138$ & 0.113 & & & \\
DBP & 0.870 & $0.747-1.014$ & 0.076 & & & \\
ALT & 0.997 & $0.991-1.004$ & 0.310 & & & \\
AST & 1.024 & $1.008-1.040$ & 0.004 & 1.040 & $1.004-1.078$ & 0.029 \\
PLT & 0.991 & $0.982-1.000$ & 0.010 & & & \\
Smoking & 1.666 & $1.187-2.338$ & 0.014 & 1.220 & $1.040-1.878$ & 0.019 \\
index & & & & & & \\
T2DM & 1.199 & $1.036-3.991$ & 0.011 & 1.054 & $1.067-3.050$ & 0.032 \\
\hline
\end{tabular}

and smoking were found to be the independent risk factors for liver fibrosis in the patients with NAFLD. Conversely, high AST level, smoking index, and diabetes mellitus were determined to be the independent risk factors for liver fibrosis in the smokers with NAFLD. These results imply that smoking is not only associated with liver fibrosis in NAFLD but also increases the risk for liver fibrosis as the smoking index increases.

The pathogenesis of NAFLD is not fully understood [1]. A widely accepted conclusion is that NAFLD is a geneticenvironment-metabolism-related disease $[1,2]$. Consumption of food high in calorie and fructose, refined carbohydrates, and sugar-sweetened beverages has been associated with NAFLD [1]. Recently, several genetic modifiers of NAFLD have been identified [25-28]. Among them, the best-characterized genetic association was found with PNPLA3, which was initially identified from genome-wide association studies and confirmed in multiple cohorts [29-32]. Liver biopsy is the gold standard for the diagnosis of NAFLD [1]. However, it cannot be routinely used because of its invasiveness. Noninvasive techniques, such as the use of FibroScan and ultrasonography, are beginning to be used for the diagnosis of NAFLD [20,22]. Their accuracy has been confirmed in many studies $[2,16,20-22]$.

The toxic and harmful substances produced by smoking can damage the antioxidant system, including cytochrome P450 and inflammatory cytokines [33]. Although the effects of smoking on CHB infection and cirrhosis have been reported $[17,34,35]$, there is limited information on the relationship between smoking and NAFLD. Hamabe et al. [19] reported that smoking is an independent risk factor for NAFLD. Suzuki et al. [18] reported that it is associated with high levels of ALT in patients with NAFLD. Herein, we found that smoking is an independent risk factor for liver fibrosis in NAFLD. For patients with NAFLD, timely smoking cessation education should be provided, and a liver fibrosis test is also necessary. However, we found a connection between smoking index and liver fibrosis among NAFLD patients. However, in this population, the smoking index and liver fibrosis grade did not show with dose response in our study. We further found that diabetes mellitus and the smoking index were the independent risk factors for fibrosis in the smokers with NAFLD. The relationship between smoking and diabetes mellitus has been well established. If smokers with NAFLD are diagnosed with diabetes mellitus, the possibility of liver fibrosis development may increase. In our study, we found that diabetes mellitus is an independent factor for liver fibrosis among NAFLD patients with smoking. Although we did not find that DM is an independent factor for liver fibrosis among all NAFLD patients, the reason may due to the relatively small patients enrolled in our study with only 12 patients diagnosed with diabetes mellitus in NAFLD without smoking. In addition, since smoking is also an independent risk factor for DM, this may impair the association of DM and liver fibrosis among all NAFLD patients.

This study has some limitations. First, the sample size of the study is relatively small. Second, the study data were collected from a single center. Given the cross-sectional nature of this study, prospective studies should be conducted to corroborate the conclusions. Multicenter clinical studies are also warranted to confirm our results for screening and early diagnosis of liver fibrosis in patients with NAFLD.

In conclusion, smoking is closely related to liver fibrosis in NAFLD. Older age, high AST level, and smoking are the independent risk factors for liver fibrosis in NAFLD. Conversely, high AST level, smoking index, and diabetes mellitus are the independent risk factors for liver fibrosis in smokers with NAFLD. For patients with NAFLD, priority screening and timely intervention should be provided if they are found to have risk factors for liver fibrosis.

\section{Data Availability}

The data used to support the findings of this study are available from the corresponding author upon request.

\section{Conflicts of Interest}

The authors declare that they have no conflicts of interest.

\section{Authors' Contributions}

Hongjie Ou and Yaojie Fu contributed equally to this work.

\section{Acknowledgments}

The authors wish to thank the nurses for their helpful assistance in the study.

\section{References}

[1] EASL, EASD, and EASO, "EASL-EASD-EASO clinical practice guidelines for the management of non-alcoholic fatty liver disease," Journal of Hepatology, vol. 64, pp. 1388-1402, 2016.

[2] N. Chalasani, Z. Younossi, J. E. Lavine et al., "The diagnosis and management of nonalcoholic fatty liver disease: practice guidance from the American Association for the Study of Liver Diseases," Hepatology, vol. 67, no. 1, pp. 328-357, 2018.

[3] I. Doycheva, K. D. Watt, and N. Alkhouri, "Nonalcoholic fatty liver disease in adolescents and young adults: the next frontier in the epidemic," Hepatology, vol. 65, no. 6, pp. 2100-2109, 2017. 
[4] I. Mikolasevic, S. Milic, L. Orlic, D. Stimac, N. Franjic, and G. Targher, "Factors associated with significant liver steatosis and fibrosis as assessed by transient elastography in patients with one or more components of the metabolic syndrome," Journal of Diabetes and Its Complications, vol. 30, no. 7, pp. 1347-1353, 2016.

[5] A. Lonardo, S. Sookoian, C. J. Pirola, and G. Targher, "Nonalcoholic fatty liver disease and risk of cardiovascular disease," Metabolism, vol. 65, no. 8, pp. 1136-1150, 2016.

[6] M. G. Radaelli, F. Martucci, S. Perra et al., "NAFLD/NASH in patients with type 2 diabetes and related treatment options," Journal of Endocrinological Investigation, vol. 41, no. 5, pp. 509-521, 2017.

[7] A. Britton, G. Mehta, D. O’Neill, and S. Bell, "Association of thirty-year alcohol consumption typologies and fatty liver: findings from a large population cohort study," Drug and Alcohol Dependence, vol. 194, pp. 225-229, 2019.

[8] I. Croci, J. S. Coombes, S. Bucher Sandbakk et al., "Non-alcoholic fatty liver disease: prevalence and all-cause mortality according to sedentary behaviour and cardiorespiratory fitness. The HUNT Study," Progress in Cardiovascular Diseases, vol. 62, no. 2, pp. 127-134, 2019.

[9] J. M. Schattenberg and I. Bergheim, "Nutritional intake and the risk for non-alcoholic fatty liver disease (NAFLD)," Nutrients, vol. 11, no. 3, p. 588, 2019.

[10] F. Lanuza, J. Sapunar, and E. Hofmann, "Análisis crítico del tratamiento de la enfermedad hepática grasa no alcohólica," Revista Médica de Chile, vol. 146, no. 8, pp. 894-901, 2018.

[11] K. Mazhar, "The future of nonalcoholic fatty liver disease treatment," Medical Clinics of North America, vol. 103, no. 1, pp. 57-69, 2019.

[12] M. Mazidi, N. Shivappa, M. D. Wirth, J. R. Hebert, and A. P. Kengne, "Diet with greater inflammatory potential is associated with higher prevalence of fatty liver among US adults," European Journal of Clinical Nutrition, vol. 1, 2018.

[13] S. Saadati, B. Hatami, Z. Yari et al., "The effects of curcumin supplementation on liver enzymes, lipid profile, glucose homeostasis, and hepatic steatosis and fibrosis in patients with non-alcoholic fatty liver disease," European Journal of Clinical Nutrition, vol. 73, no. 3, pp. 441-449, 2019.

[14] Y. Mu, B. J. Patters, N. M. Midde, H. He, S. Kumar, and T. J. Cory, "Tobacco and antiretrovirals modulate transporter, metabolic enzyme, and antioxidant enzyme expression and function in polarized macrophages," Current HIV Research, vol. 16, no. 5, pp. 354-363, 2018.

[15] S. a. Munther, "The effects of cigarette smoking and exercise on total salivary antioxidant activity," The Saudi Dental Journal, vol. 31, no. 1, pp. 31-38, 2019.

[16] S. Cai, Z. Ou, D. Liu et al., "Risk factors associated with liver steatosis and fibrosis in chronic hepatitis B patient with component of metabolic syndrome," United European Gastroenterology Journal, vol. 6, no. 4, pp. 558-566, 2018.

[17] M. Xiong, J. Li, S. Yang et al., "Impacts of cigarette smoking on liver fibrosis and its regression under therapy in male patients with chronic hepatitis B," Liver International, vol. 39, no. 8, 2019.

[18] A. Suzuki, K. Lindor, J. S. Saver et al., "Effect of changes on body weight and lifestyle in nonalcoholic fatty liver disease," Journal of Hepatology, vol. 43, no. 6, pp. 1060-1066, 2005.

[19] A. Hamabe, H. Uto, Y. Imamura et al., "Impact of cigarette smoking on onset of nonalcoholic fatty liver disease over a 10year period," Journal of Gastroenterology, vol. 46, no. 6, pp. 769-778, 2011.

[20] V. d. Lédinghen, G. L.-H. Wong, J. Vergniol et al., "Controlled attenuation parameter for the diagnosis of steatosis in non-alcoholic fatty liver disease," Journal of Gastroenterology and Hepatology, vol. 31, no. 4, pp. 848-855, 2016.

[21] H. Ou, S. Cai, Y. Liu, M. Xia, and J. Peng, "A noninvasive diagnostic model to assess nonalcoholic hepatic steatosis in patients with chronic hepatitis B," Therapeutic Advances in Gastroenterology, vol. 10, no. 2, pp. 207-217, 2017.

[22] M. Sasso, S. Audière, A. Kemgang et al., "Liver steatosis assessed by controlled attenuation parameter (CAP) measured with the XL probe of the FibroScan: a pilot study assessing diagnostic accuracy," Ultrasound in Medicine and Biology, vol. 42, no. 1, pp. 92-103, 2016.

[23] X. Xue and S. Cai, "Comment on "assessment of liver stiffness in pediatric fontan patients using transient elastography"," Canadian Journal of Gastroenterology and Hepatology, vol. 2016, Article ID 9343960, 2016.

[24] J. Zeng, S. Cai, J. Liu, X. Xue, X. Wu, and C. Zheng, “Dynamic changes in liver stiffness measured by transient elastography predict clinical outcomes among patients with chronic hepatitis B," Journal of Ultrasound in Medicine, vol. 36, no. 2, pp. 261-268, 2017.

[25] Q. M. Anstee, G. Targher, and C. P. Day, "Progression of NAFLD to diabetes mellitus, cardiovascular disease or cirrhosis," Nature Reviews Gastroenterology and Hepatology, vol. 10, no. 6, pp. 330-344, 2013.

[26] L. Valenti, A. Alisi, E. Galmozzi et al., "I148M patatin-like phospholipase domain-containing 3 gene variant and severity of pediatric nonalcoholic fatty liver disease," Hepatology, vol. 52, no. 4, pp. 1274-1280, 2010.

[27] L. Valenti, A. Al-Serri, A. K. Daly et al., "Homozygosity for the patatin-like phospholipase-3/adiponutrin I148M polymorphism influences liver fibrosis in patients with nonalcoholic fatty liver disease," Hepatology, vol. 51, no. 4, pp. 1209-1217, 2010.

[28] L. Valenti and P. Dongiovanni, "Mutant PNPLA3 I148M protein as pharmacological target for liver disease," Hepatology, vol. 66, no. 4, pp. 1026-1028, 2017.

[29] R. Aller, C. Laserna, M. A. Rojo et al., "Role of the PNPLA3 polymorphism rs738409 on silymarin + vitamin E response in subjects with non-alcoholic fatty liver disease," Revista Española de Enfermedades Digestivas, vol. 110, pp. 634-640, 2018.

[30] L. J. McGeoch, P. R. Patel, and J. P. Mann, "PNPLA3: a determinant of response to low-fructose diet in nonalcoholic fatty liver disease," Gastroenterology, vol. 154, no. 4, pp. 1207-1208, 2018.

[31] S. Pelusi, A. Cespiati, R. Rametta et al., "Prevalence and risk factors of significant fibrosis in patients with nonalcoholic fatty liver without steatohepatitis," Clinical Gastroenterology and Hepatology, vol. 17, no. 11, pp. 2310-2319.e6, 2019.

[32] N. Stefan, H.-U. Häring, and K. Cusi, "Non-alcoholic fatty liver disease: causes, diagnosis, cardiometabolic consequences, and treatment strategies," The Lancet Diabetes and Endocrinology, vol. 7, no. 4, pp. 313-324, 2019.

[33] W. C. Ou, Y. C. Huang, C. L. Huang et al., "Interaction between cytochrome P450 2A6 and Catechol-O-Methyltransferase genes and their association with smoking risk in young men," Behavioral and Brain Functions, vol. 13, p. 8, 2017.

[34] K. S. Shohdy and O. Abdel-Rahman, "Is smoking causallyassociated with hepatitis B virus-related hepatocellular carcinoma?," Annals of Translational Medicine, vol. 7, no. S1, p. S44, 2019.

[35] Y. H. Wang, Y. H. Chuang, C. F. Wu et al., "Smoking and hepatitis B virus-related hepatocellular carcinoma risk: the mediating roles of viral load and alanine aminotransferase," Hepatology, vol. 69, no. 4, pp. 1412-1425, 2019. 


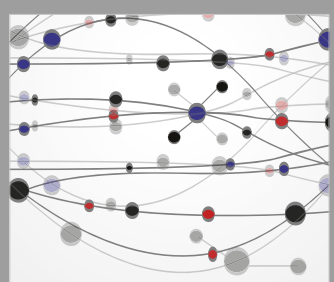

The Scientific World Journal
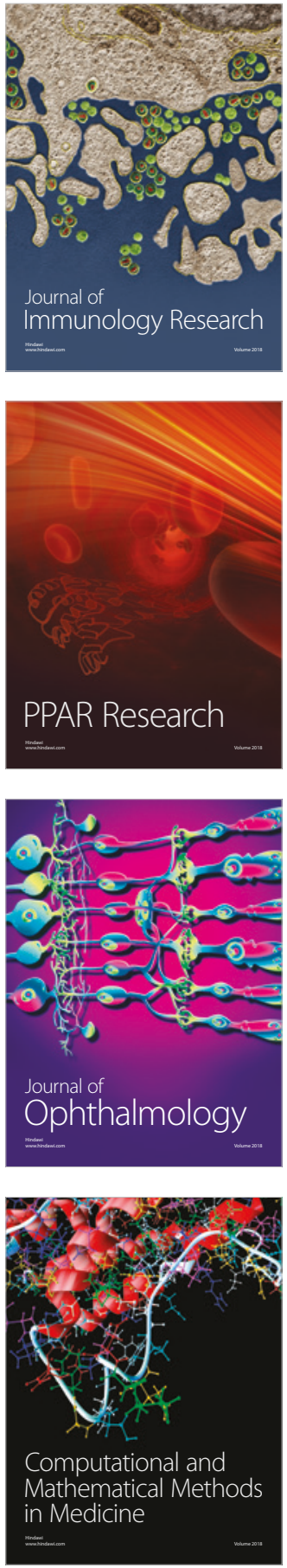

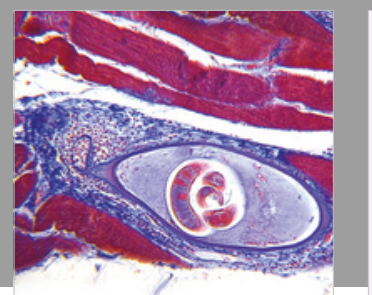

Gastroenterology Research and Practice

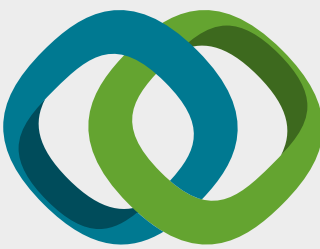

\section{Hindawi}

Submit your manuscripts at

www.hindawi.com
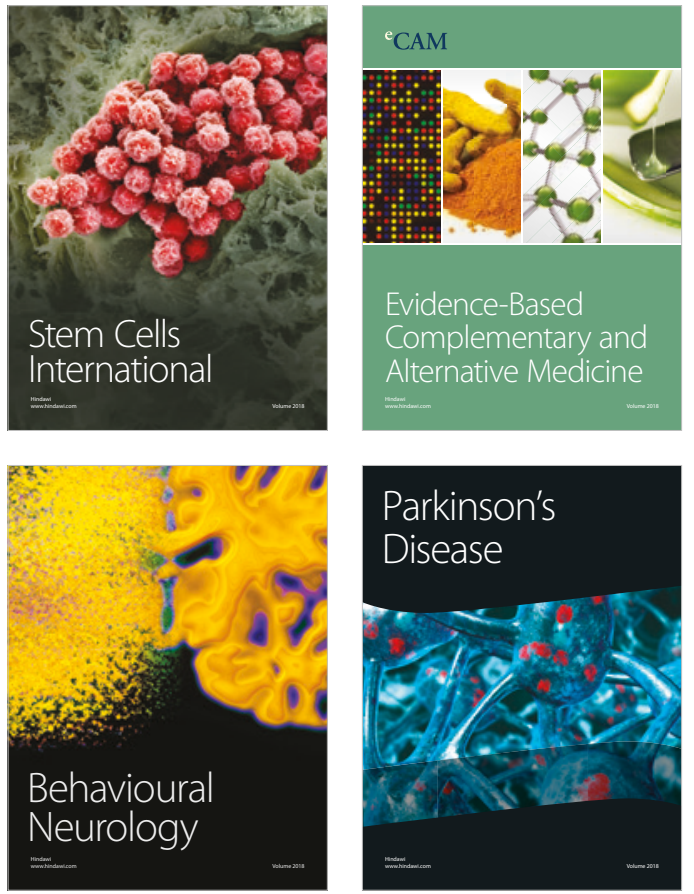

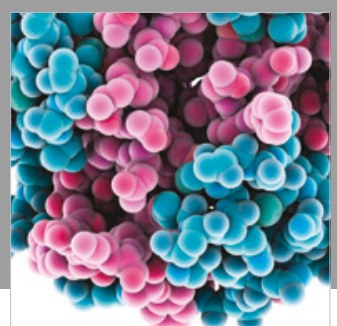

ournal of

Diabetes Research

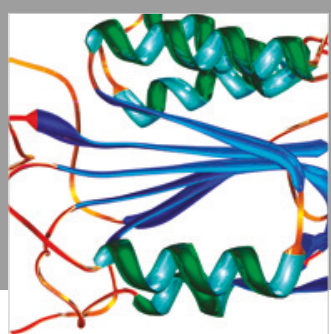

Disease Markers
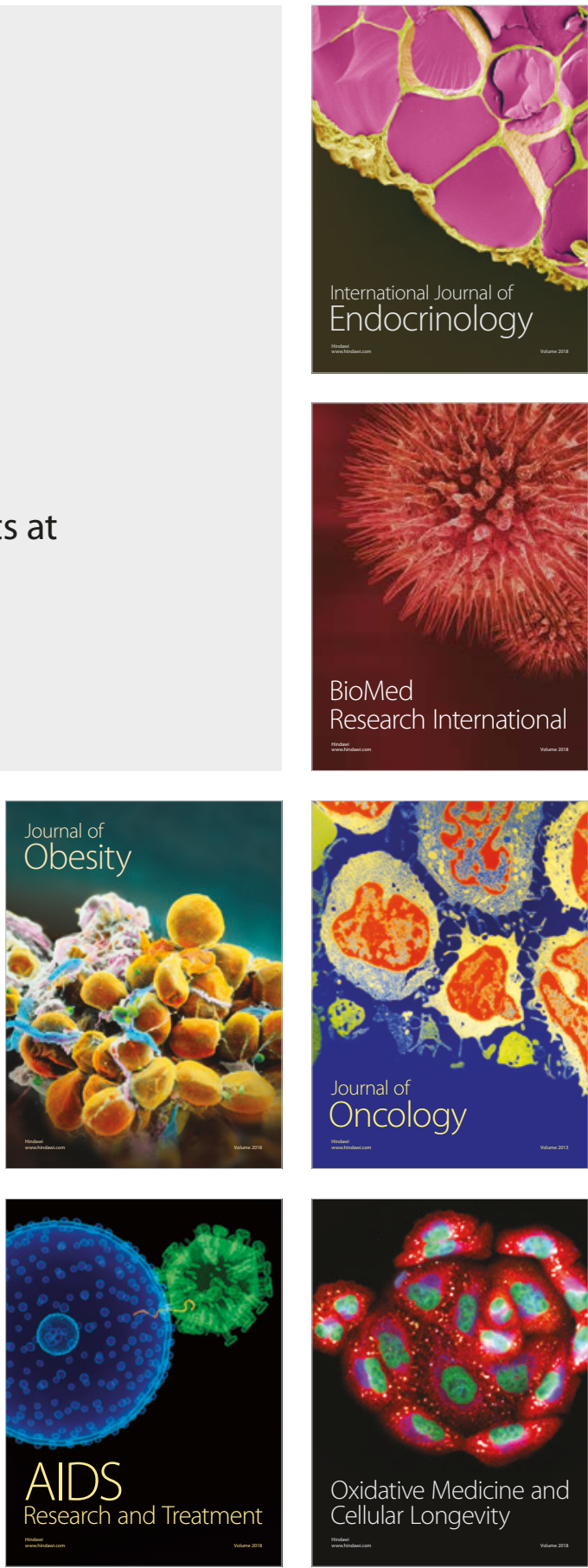